|| Print ISSN: 2589-7837 || Online ISSN: 2581-3935 ||

International Journal of Medical Science and Diagnosis Research (IJMSDR)

Available Online at www.ijmsdr.com

NLM (National Library of Medicine ID: 101738824)

Case Study

Volume 5, Issue 1; January: 2021; Page No. 39-42

\title{
FIBROUS ANKYLOSIS OF TEMPOROMANDIBULAR JOINT: PRESENTING WITH SUDDEN ONSET COMPLETE INABILITY TO OPEN MOUTH.
}

\section{Dr Rupesh Kumar Namdev}

Consultant Radiologist, Innorad Healthcare, Bhilai, Chhattisgarh, India

Conflicts of Interest: Nil

Corresponding author: Dr Rupesh Kumar Namdev

DOI: https://doi.org/10.32553/ijmsdr.v5i1.740

\begin{abstract}
:
The temporomandibular joint makes it possible to open and close your mouth, as involving chewing, swallowing, speaking, and yawning activity. Ankylosis of the Temporomandibular joint which includes partial or complete fusion of the joint with resulting restricted opening of mouth, restricted mandibular movements (hypomobility) with deviation to the affected side on opening of the mouth. The pathology is multifactorial and restoration of function usually involves surgical intervention and dependence on the exact pathology situated at the root of pons. It is essential to evaluate the underlying pathology, its degree for proper planning of surgery and follow-up. 3-D CT scan of facial bones provide a reliable and consistent diagnosis and evaluation for temporomandibular joint ankylosis.
\end{abstract}

Keywords: TMJ, ankylosis, 3-D CT scan, facial asymmetry.

\section{Introduction:}

A 24 year old female presented with sudden onset complete inability to open mouth. She was having restricted jaw movement since childhood and also had difficulty chewing larger bites of food. There was no history of tobacco abuse. No history of relevant trauma.

On examination it was observed that there was restricted mouth opening. The face was deviated to the right side. Rest of the facial profile was unremarkable. No ulcers in mouth, no lymphadenopathy. Dentition visualized appeared normal.
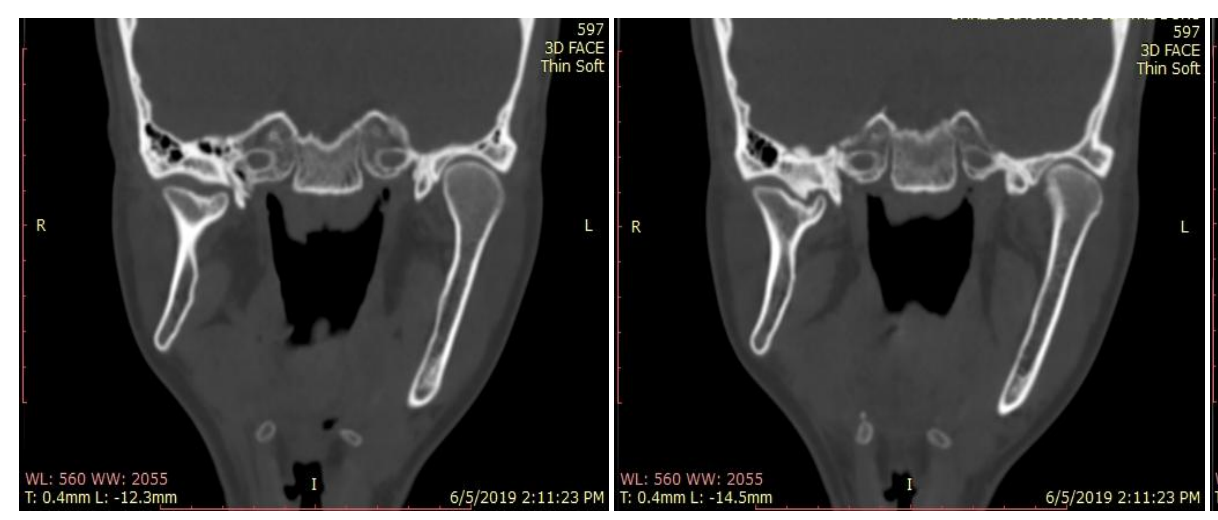

\section{Diagnosis / differential diagnosis}

The differential diagnosis for restricted mouth includes - temporomandibular ankylosis, TMJ dislocation, submucosal fibrosis, mandibular fracture, and facial palsy.

\section{Investigations and interventions}

The patient was referred to for CT scan of Facial bone without contrast.

The key images are shown below: 


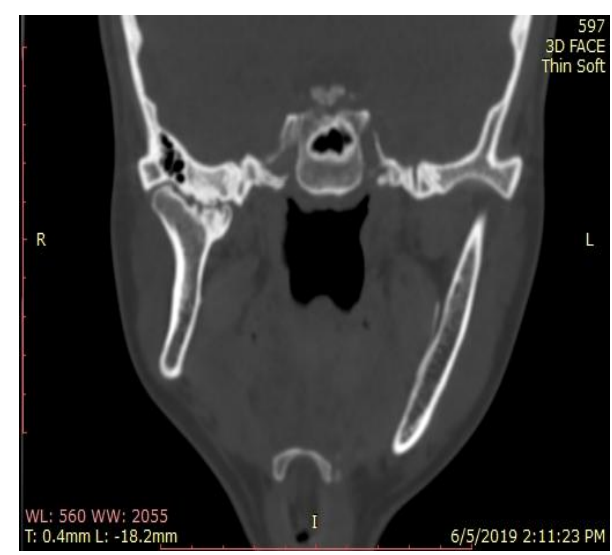

Figure 1 a-d: coronal sections
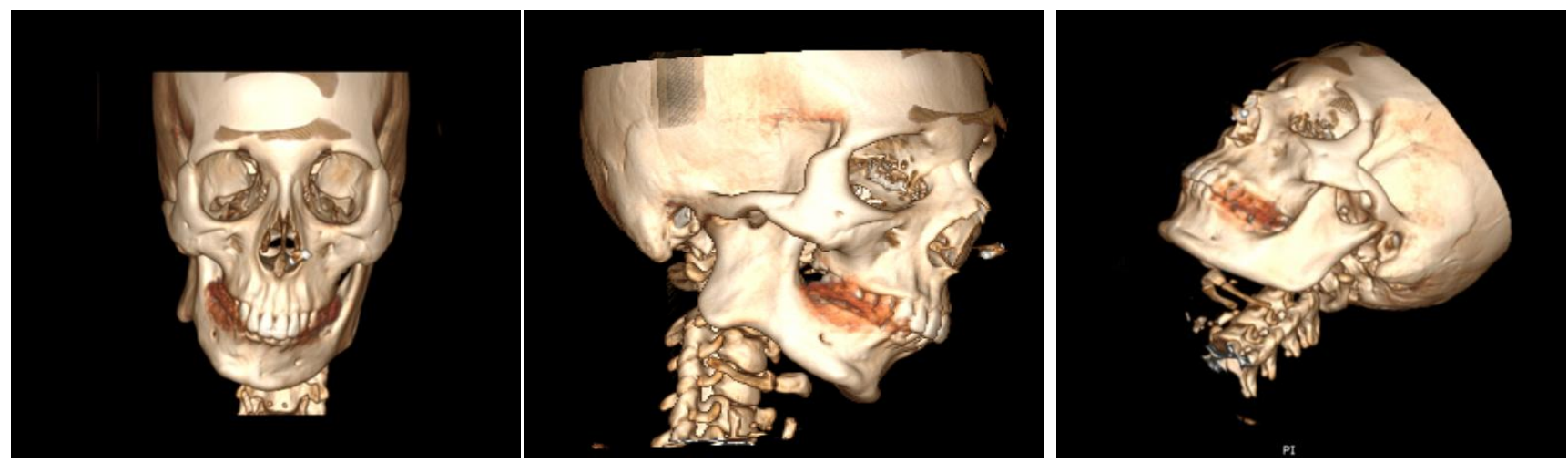

Figure 2 a $-\mathbf{c}: 3 d$ reconstruction

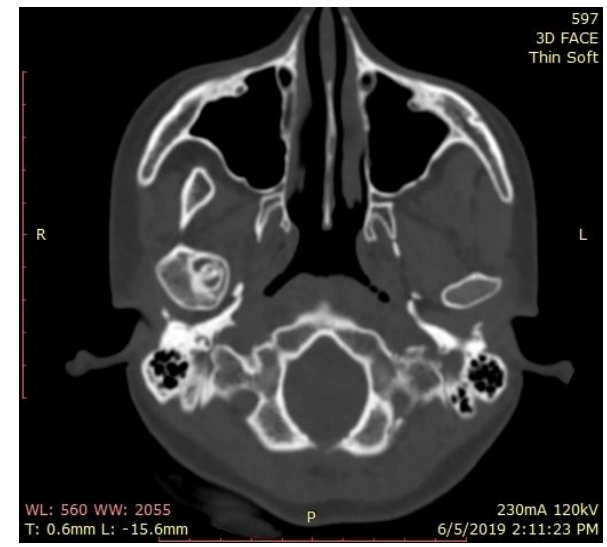

Figure 3: axial section of right TMJ

Figure 1-3: There is flattening, and deformity noted involving the right mandibular condyle with flattening of the right temporomandibular fossa with irregularity of cortex and subchondral sclerosis. No evidence of complete bony fusion. Prominent antigonial notch of mandible on right side. The vertical length of right mandibular ramus is shorter than the left ramus.

Findings are suggestive of Sawhey type I/ fibrous ankylosis of the right temporomandibular joint. Left TMJ appears unremarkable. 


\section{Treatment:}

The mainstay of treatment is surgical. Aims and Objectives of surgery are to create a mobile joint by resection of ankylosed mass and creation of a gap, to reconstruct the joint and restore the vertical height of the ramus, and restore normal facial growth pattern

The procedures include condylectomy, gap arthroplasty and interpositional arthroplasty.

\section{Discussion}

Ankylosis is defined as the pathological partial or complete fusion of a joint resulting in restricted movement across the joint.

Ankylosis of the Temporomandibular joint causes restricted opening of mouth, restricted mandibular movements (hypomobility) with deviation to the affected side on opening of the mouth.

Ankylosis of the Temporomandibular joint can be seen at any age group but is common in adolescent

Ankylosis of the Temporomandibular joint can be unilateral or bilateral, fibrous, or bony, intra-articular or extra-articular, complete, or partial ankylosis

Common causes include perinatal trauma associated with forcep delivery, post-natal trauma including mandibular condyle fracture, Infections and Inflammatory diseases like rheumatoid arthritis, septic arthritis, otomastoiditis and degenerative arthritis of TM joints.

Systemic disease like Smallpox, seronegative spondyloarthropathy, Syphilis and typhoid also are known to be causative in some cases.

\section{Clinical features}

- ipsilateral chin deviation and facial deformity

- Inability to open the jaws and absent condylar movements on affected side

\section{Radiographic features}

- Partial or complete fibrous or bony fusion of joint with obliterated joint space

- Prominent antigonial notch

- Coronoid hyperplasia

- Short vertical ramus on the affected side

There are many classification systems but the commonly referred classification was given by Sawhney CP in 1986.
Type 1 - fibrous ankylosis. There is flattening and deformity at TM fossa and mandibular condyle.

Type 2 - bony ankylosis with sparing of central part.

Type 3 - bony bridging between mandibular condyle and zygomatic arch.

Type 4 - complete fusion of joint which appears replaced by single bony mass

The mainstay of treatment is surgical. Aims and Objectives of surgery are to create a mobile joint by resection of ankylosed mass and creation of a gap, to reconstruct the joint and restore the vertical height of the ramus, and restore normal facial growth pattern

The procedures include condylectomy, gap arthroplasty and interpositional arthroplasty.

\section{Conclusion}

Ankylosis of the Temporomandibular joint is the pathological partial or complete fusion of the joint and causes restricted opening of mouth, restricted mandibular movements (hypomobility) with deviation to the affected side on opening of the mouth.

Common causes include perinatal trauma associated with forcep delivery, post-natal trauma including mandibular condyle fracture, Infections and Inflammatory diseases like rheumatoid arthritis, septic arthritis, otomastoiditis and degenerative arthritis of TM joints. Systemic disease like Smallpox, seronegative spondyloarthropathy, Syphilis and typhoid also are known to be causative in some cases.

The mainstay of treatment is surgical. to create a mobile joint by resection of ankylosed mass and creation of a gap, to reconstruct the joint and restore the vertical height of the ramus and restore normal facial growth pattern.

Imaging studies demonstrate partial or complete fibrous or bony fusion of joint with obliterated joint space, prominent antigonial notch, coronoid hyperplasia, and short vertical ramus on the affected side.

Imaging plays an important role in diagnosis, quantification, planning of surgery and follow up to see recurrence.

\section{References}

1. Jaju, S. R., Chhatrapati, S., Ubale, P., \& Ingle, S. (2018). Anaesthetic Management of a Paediatric Patient with Bilateral Temporomandibular Joint Ankylosis: A Case Report. International Journal 
of Contemporary Medical Research [IJCMR], 5(4). doi:10.21276/ijcmr.2018.5.4.42

2. Suragimath, A., Suragimath, G., \& Devendrappa, S. (2016). Temporomandibular joint ankylosis. Journal of Indian Academy of Oral Medicine and Radiology, 28(3), 334. doi:10.4103/09721363.195646

3. Thompson, J. R., Christiansen, E., Hasso, A. N.,
\& Hinshaw, D. B. (1984). Temporomandibular joints: High-resolution computed tomographic evaluation. Radiology, 150(1), 105-110. doi:10.1148/radiology.150.1.6689750

4. Sawhney, C. P. (1986). Bony Ankylosis of Temporomandibular Joint. Plastic and Reconstructive Surgery, 77(1), 39. doi:10.1097/ 00006534-198601000-00007 